\section{Atrkivoc

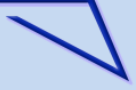

Archive for

Organic Chemistry
The Free Internet Journal

for Organic Chemistry
Paper

Arkivoc 2017, part v, 20-31

\title{
Ultrasound-assisted, $\mathrm{ZnBr}_{2}$-catalyzed regio- and stereoselective synthesis of
} novel 3,3'-dispiropyrrolidine bisoxindole derivatives via 1,3-dipolar cycloaddition reaction of an azomethine ylide

\author{
Mostafa Kiamehr, ${ }^{\text {a,* }}$ Mohammad R. Khodabakhshi, ${ }^{b}$ Firouz M. Moghaddam, ${ }^{c}$ \\ Alexander Villinger, ${ }^{d}$ and Peter Langer ${ }^{d, e}$ \\ a Department of Chemistry, Faculty of Science, University of Qom, Ghadir Blvd, Qom, Iran \\ ${ }^{b}$ Applied Biotechnology Research Center, Baqiyatallah University of Medical Sciences, Tehran, Iran \\ c Laboratory of Organic Synthesis and Natural Products, Department of Chemistry, \\ Sharif University of Technology, P. O. Box 11155-9516 Tehran, Iran \\ ${ }^{d}$ Institut für Chemie der Universität Rostock, Albert-Einstein-Strasse 3a, D-18059 Rostock, Germany \\ e Leibniz Institut für Katalyse an der Universität Rostock, Albert-Einstein-Strasse 29a, \\ D-18059 Rostock, Germany \\ Email: mkiamehr@yahoo.com m.kiamehr@qom.ac.ir
}

Received 02-02-2017

Accepted 03-14-2017

Published on line 06-27-2017

\section{Abstract}

Ultrasound irradiation in presence of $20 \% \mathrm{ZnBr}_{2}$ effectively promotes regio- and stereo-selective cycloaddition reaction of azomethine ylide with a series of (E)-3-benzylideneindolin-2-ones to afford 3,3'-dispiropyrrolidine bisoxindole derivatives in excellent yields in methanol at room temperature. The factors affecting the cycloaddition reaction, for example solvent, catalyst, ultrasonic irradiation, are examined in detail to find the mildest conditions and highest reaction yields. The structure and stereochemistry of cycloadducts were determined by spectroscopic data and confirmed by X-ray crystallographic analysis.
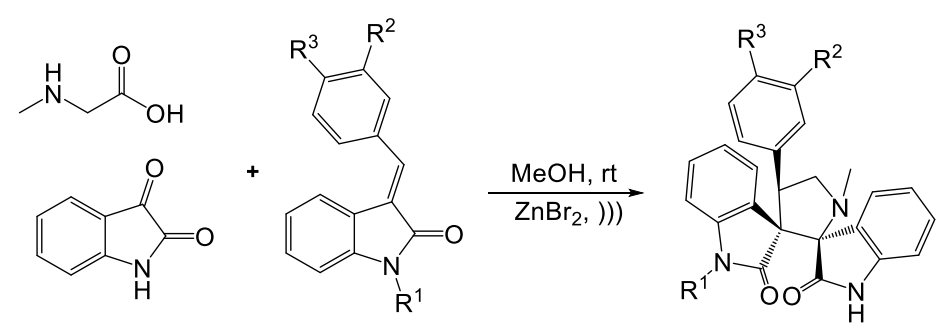

Keywords: Ultrasonic irradiation, azomethine ylide, 1,3-dipolar cycloaddition, dispiro oxindole, $\mathrm{ZnBr}_{2}$ 
The 1,3-dipolar cycloaddition reaction of azomethine ylides is a powerful tool for the construction of various types of complex polyheterocyclic frameworks. ${ }^{1-3}$ In recent years the azomethine ylide has gained a vital place in the synthesis of heterocyclic compounds as it serves as an important building block for the construction of nitrogen-containing five membered heterocycles, ${ }^{4-8}$ which are often an integral part of many natural products and bioactive molecules. ${ }^{9,10}$ The 3,3'-spirocyclooxindole skeleton is found in a growing number of natural or synthetic products presenting various biological activities. ${ }^{11-13}$ In particular, the spiropyrrolidinyloxindole, due to its presence in a large number of bioactive alkaloids, such as coerulescine ${ }^{14,15}(\mathbf{1})$, inhibitor of the MDM2-

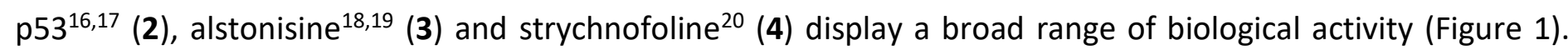
Some spiropyrrolidines are potential antileukaemic and anticonvulsant agents ${ }^{21}$ and possess antiviral and local anaesthetic activities, ${ }^{15}$ and this has attracted considerable attention from organic chemists. The challenges associated with the synthesis of spiro- or dispiro-heterocycles containing the 3,3'-pyrrolidinyl-spirooxindole core have made them the subject of several elegant synthetic investigations. ${ }^{22-32}$

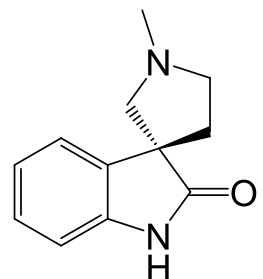

coerulescine (1)

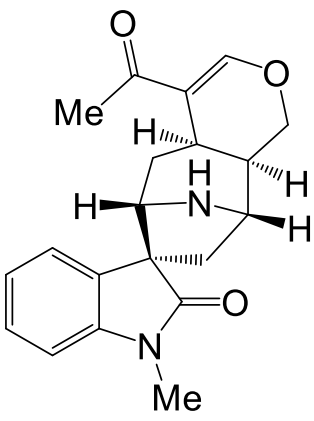

alstonisine (3)

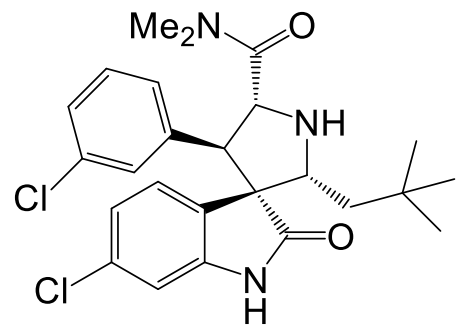

inhibitor of the MDM2-p53 (2)

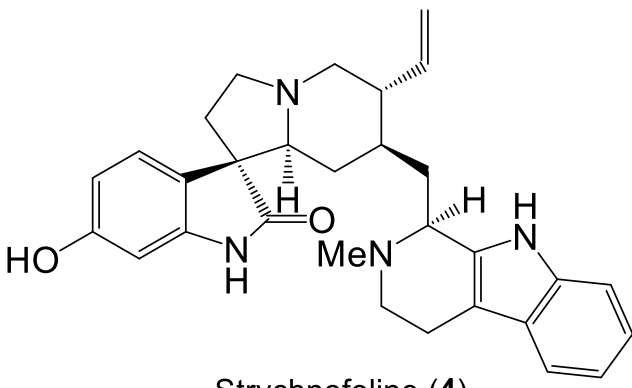

Strychnofoline (4)

Figure 1. Biologically important molecules containing spiropyrrolidinyloxindole skeleton.

Ultrasound irradiation has increasingly been used as a powerful tool for the preparation of organic molecules either in homogeneous or in heterogeneous liquid reaction systems. ${ }^{33,34}$ The success and advantages of this method include shorter reaction time, higher yield, higher product purity, improved selectivity, reduced side product formation, and use of milder reaction conditions when compared with conventional heating. ${ }^{35,36}$ Therefore, recently, ultrasonic irradiation has received considerable attention of researchers and numerous examples under this condition for constructing heterocycles with interesting properties have been reported. ${ }^{37-39}$ Zinc halides, particularly $\mathrm{ZnBr}_{2}$, are very interesting catalysts due to their low toxicity, low cost, ease of handling, stability in air and water, recoverability and use in numerous chemical transformations. ${ }^{40}$ 
In continuation of our interest in the cycloaddition reaction to synthesis of novel polycyclic nitrogen heterocycles ${ }^{41-43}$ and as part of our concern in the using catalyst, ${ }^{44,45}$ herein we report an efficient, highly atom-economic and regioselective synthesis of novel 3,3'-dispiropyrrolidine bisoxindoles 8a-I via the one-pot, three-component condensation of azomethine ylide (generated in situ from sarcosine $\mathbf{5}$ and isatin 6) with the Knoevenagel adduct (E)-3-benzylideneindolin-2-ones 7a-I (Scheme 1). The reaction is performed under ultrasound irradiation, in the presence of $20 \% \mathrm{ZnBr}_{2}$ as a catalyst in methanol at ambient temperature.

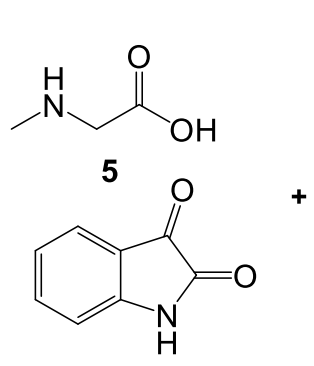

6

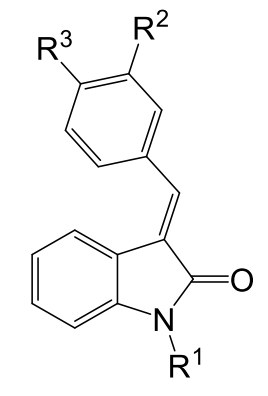

7a-I

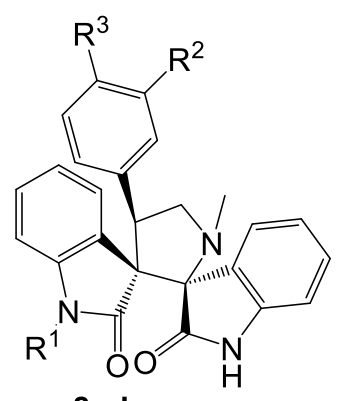

$8 a-I$

Scheme 1. Synthesis of 3,3'-dispiropyrrolidine bisoxindole derivatives 8 a-I.

\section{Results and Discussion}

The (E)-3-benzylideneindolin-2-ones 7a-I were prepared according to the reported procedure via condensation of indolin-2-ones $\mathbf{9}$ and various aromatic aldehydes $\mathbf{1 0}$ with the predominant formation of $E$ isomer (Scheme 2). ${ }^{46}$ These diastereomers were separated by column chromatography to give pure compounds (E) and (Z)-3benzylideneindolin-2-ones $\mathbf{7}$ and $\mathbf{1 1}$ in good yields. The structure of the isomers was deduced on the basis of ${ }^{1} \mathrm{H}$ NMR spectral data. ${ }^{47,48}$

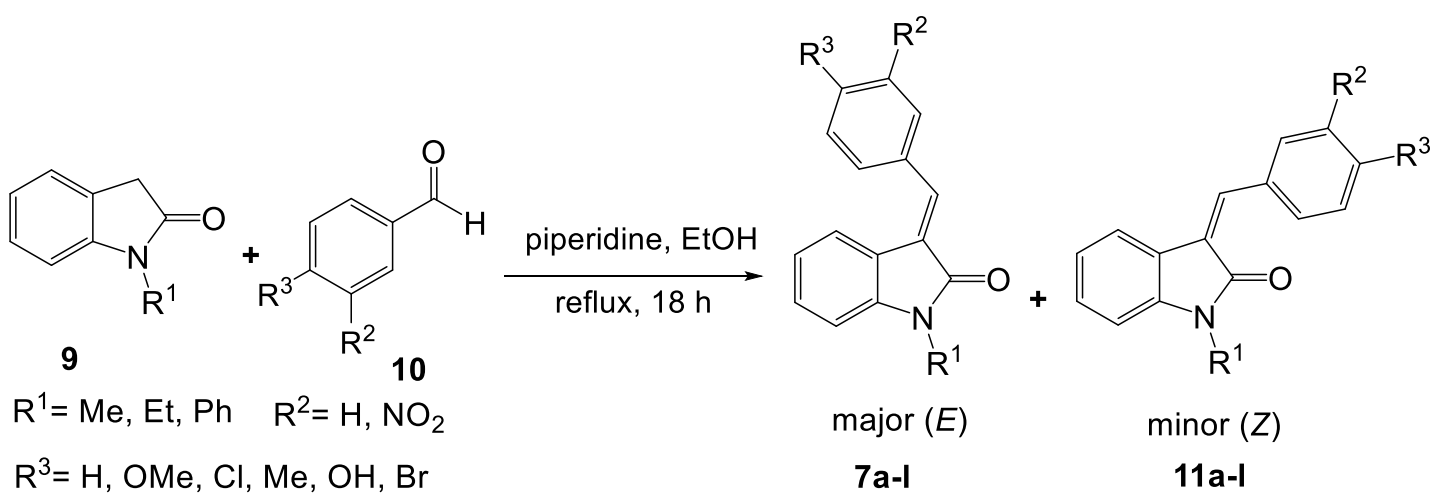

Scheme 2. Synthesis of $(E)$ and (Z)-3-benzylideneindolin-2-ones.

We studied the effect of solvents, catalysts and ultrasound irradiation on cycloaddition reaction of the 1,3dipole generated from isatin 6 and sarcosine $\mathbf{5}$ with the Knoevenagel adduct (E)-3-benzylidene-indolin-2-one 7a (Table 1). Initially a series of solvents were investigated to find the best reaction conditions (Entries 1-7). Among the solvents used, methanol at $65^{\circ} \mathrm{C}$ (reflux temp.) was found to be the best one in terms of highest yield and shortest reaction time (Entry 7$)$. Then this reaction was examined in some different catalysts under refluxing methanol. The results showed that $\mathrm{ZnBr}_{2}$ is the best catalyst to achieve the desired product in the highest yield and the shortest reaction time (Entry 11). Increasing and reducing the amount of $\mathrm{ZnBr}_{2}$ from 100 

mol\% to 20 mol\% did not have any effect on the yield of reaction (Entries 13 and 14), but reducing the amount of $\mathrm{ZnBr}_{2}$ to $10 \mathrm{~mol} \%$ shows little negative effect (Table 1, entry 15). To evaluate the effects of ultrasound irradiation on the reactivity of reagents, this reaction was performed in methanol and $\mathrm{ZnBr}_{2}(20$ mol\%) under ultrasound irradiation conditions at room temperature (Table 1, entry 16). The 1,3-dipole generated from isatin 6 and sarcosine 5 reacted readily with the adduct (E)-3-benzylideneindolin-2-one 7a under ultrasound irradiation to give 3,3'-dispiropyrrolidine bisoxindole 8 a in $98 \%$ yield through an intermolecular 1,3-dipolar cycloaddition reaction with high regio- and stereoselectivity in presence of $20 \% \mathrm{ZnBr}_{2}$ in methanol within 30 minute. The reaction produced a single product in all cases, as which was confirmed by TLC and $1 \mathrm{H}$ NMR spectral data. Comparison of $\mathrm{ZnBr}_{2}$ catalyzed ultrasonic irradiation and classical reactions showed that the reaction time is reduced from 90 minutes to only 30 minutes by ultrasonic irradiation at room temperature, which indicates that the ultrasonic irradiation plays an important role in the rate and yield enhancement.

Table1. Effect of solvent, catalyst and ultrasound irradiation on the yield of product $\mathbf{8 a}$

\begin{tabular}{cccccc}
\hline Entry & Solvent & Catalyst & Temp. $\left({ }^{\circ} \mathrm{C}\right)$ & Time $(\mathrm{min})$ & Yield $^{a}(\%)$ \\
\hline 1 & $\mathrm{CH}_{3} \mathrm{CN}$ & - & Reflux & 180 & 50 \\
2 & Toluene & - & Reflux & 300 & 14 \\
3 & Benzene & - & Reflux & 300 & 25 \\
4 & $1,4-$ Dioxane & - & Reflux & 300 & 50 \\
5 & $\mathrm{CH}_{3} \mathrm{CH}_{2} \mathrm{OH}$ & - & Reflux & 180 & 60 \\
6 & $\mathrm{CH}_{3} \mathrm{OH}$ & - & rt & 300 & 10 \\
7 & $\mathrm{CH}_{3} \mathrm{OH}$ & - & Reflux & 180 & 82 \\
8 & $\mathrm{CH}_{3} \mathrm{OH}$ & $\mathrm{SiO}_{2}(50 \mathrm{~mol} \%)$ & Reflux & 90 & 88 \\
9 & $\mathrm{CH}_{3} \mathrm{OH}$ & $\mathrm{ZnCl}_{2}(50 \mathrm{~mol} \%)$ & Reflux & 90 & 84 \\
10 & $\mathrm{CH}_{3} \mathrm{OH}$ & $\mathrm{ZrOCl}_{2}(50 \mathrm{~mol} \%)$ & Reflux & 90 & 85 \\
11 & $\mathrm{CH}_{3} \mathrm{OH}$ & $\mathrm{ZnBr}_{2}(50 \mathrm{~mol} \%)$ & Reflux & 90 & 92 \\
12 & $\mathrm{CH}_{3} \mathrm{OH}$ & $\mathrm{ZnO}^{2}(50 \mathrm{~mol} \%)$ & Reflux & 90 & 82 \\
13 & $\mathrm{CH}_{3} \mathrm{OH}$ & $\mathrm{ZnBr}_{2}(100 \mathrm{~mol} \%)$ & Reflux & 90 & 92 \\
14 & $\mathrm{CH}_{3} \mathrm{OH}$ & $\mathrm{ZnBr}_{2}(20 \mathrm{~mol} \%)$ & Reflux & 90 & 92 \\
15 & $\mathrm{CH}_{3} \mathrm{OH}$ & $\mathrm{ZnBr}_{2}(10 \mathrm{~mol} \%)$ & Reflux & 90 & 87 \\
$16^{b}$ & $\mathrm{CH}_{3} \mathrm{OH}$ & $\mathrm{ZnBr}_{2}(20 \mathrm{~mol} \%)$ & rt & 30 & 98 \\
\hline
\end{tabular}

${ }^{a}$ Isolated product. ${ }^{b}$ This reaction was conducted under ultrasound irradiation.

The scope of the reaction was extended by reacting various (E)-3-benzylideneindolin-2-ones 7a-I with azomethine ylide generated from isatin 6, and sarcosine 5 to give 3,3'-dispiropyrrolidine bisoxindole derivatives $\mathbf{8 a - 1}$ in excellent yields (Table 2). The reaction proceeded smoothly and a single regioisomer was isolated in all the cases studied.

The structures and regiochemistry of cycloadducts $8 \mathrm{a}-\mathrm{I}$ were characterized by $\mathrm{IR},{ }^{1} \mathrm{H} /{ }^{13} \mathrm{C} \mathrm{NMR}, \mathrm{GCMS}$ and HRMS data. For instance, the IR spectrum of cycloadduct 8a showed three characteristic peaks at 1698 (for other derivatives are separated) and $3168 \mathrm{~cm}^{-1}$ corresponding to the two oxindole ring carbonyls and the secondary amide NH groups, respectively. In the ${ }^{1} \mathrm{H}$ NMR spectrum of 8 a two singlets appeared at $\delta 2.30$ and 
Table 2. Structures of synthesized compounds $8 \mathrm{a}--^{a}$

\begin{tabular}{cccccc}
\hline Entry & $\mathrm{R}^{1}$ & $\mathrm{R}^{2}$ & $\mathrm{R}^{3}$ & Product & Yield $^{b}(\%)$ \\
\hline 1 & $\mathrm{CH}_{3}$ & $\mathrm{H}$ & $\mathrm{H}$ & $\mathbf{8 a}$ & 98 \\
2 & $\mathrm{CH}_{3}$ & $\mathrm{H}$ & $\mathrm{OCH}_{3}$ & $\mathbf{8 b}$ & 90 \\
3 & $\mathrm{CH}_{3}$ & $\mathrm{H}$ & $\mathrm{Cl}$ & $\mathbf{8 c}$ & 92 \\
4 & $\mathrm{CH}_{3}$ & $\mathrm{NO}_{2}$ & $\mathrm{H}$ & $\mathbf{8 d}$ & 88 \\
5 & $\mathrm{C}_{2} \mathrm{H}_{5}$ & $\mathrm{NO}_{2}$ & $\mathrm{H}$ & $\mathbf{8 e}$ & 85 \\
6 & $\mathrm{C}_{2} \mathrm{H}_{5}$ & $\mathrm{H}$ & $\mathrm{CH}_{3}$ & $\mathbf{8 f}$ & 92 \\
7 & $\mathrm{C}_{2} \mathrm{H}_{5}$ & $\mathrm{H}$ & $\mathrm{Cl}$ & $\mathbf{8 g}$ & 95 \\
8 & $\mathrm{C}_{2} \mathrm{H}_{5}$ & $\mathrm{H}$ & $\mathrm{OH}$ & $\mathbf{8 h}$ & 88 \\
9 & $\mathrm{C}_{2} \mathrm{H}_{5}$ & $\mathrm{H}$ & $\mathrm{Br}$ & $\mathbf{8 i}$ & 97 \\
10 & $\mathrm{C}_{6} \mathrm{H}_{5}$ & $\mathrm{H}$ & $\mathrm{CH}_{3}$ & $\mathbf{8 j}$ & 90 \\
11 & $\mathrm{C}_{6} \mathrm{H}_{5}$ & $\mathrm{H}$ & $\mathrm{OCH}_{3}$ & $\mathbf{8 k}$ & 86 \\
12 & $\mathrm{C}_{6} \mathrm{H}_{5}$ & $\mathrm{H}$ & $\mathrm{Cl}$ & $\mathbf{8 l}$ & 94 \\
\hline
\end{tabular}

${ }^{a}$ All the reactions were conducted under ultrasound irradiation, using 20 mol\% $\mathrm{ZnBr}_{2}$ in methanol at room temperature for 30 minute. ${ }^{b}$ Isolated product.

2.96 for two $-\mathrm{NCH}_{3}$ protons of the pyrrolidine ring and oxindole ring, respectively. The $-\mathrm{NCH}_{2}$ protons and benzylic proton of pyrrolidine ring appeared as multiplet and triplet at $\delta$ 4.47-4.53 and 3.73 which explained the regiochemistry of the cycloaddition. In contrast, if the other regionisomer had been formed, the benzylic proton would have appeared as a singlet in the ${ }^{1} \mathrm{H} N M R$ spectrum. The $-\mathrm{NH}$ proton of the oxindole showed a broad singlet at $\delta 7.93 \mathrm{ppm}$. For the ${ }^{13} \mathrm{C}$ NMR spectrum of $\mathbf{8 a}$, the two spiro quaternary carbons resonated at 64.9, $79.5 \mathrm{ppm}$ and the oxindoles carbonyl carbons resonated at 177.5 and 179.0 ppm. Finally the regiochemistry and formation of the products $\mathbf{8}$ was confirmed by the structure determined from an X-ray crystallographic study of the single crystal of $8 \mathbf{i}$ (Figure 2). ${ }^{49}$

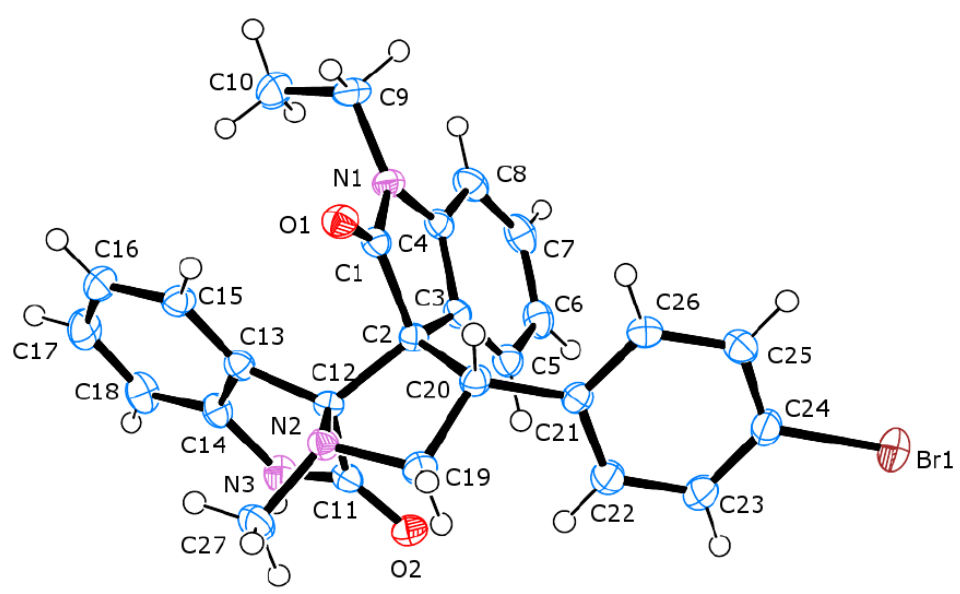

Figure 2. ORTEP diagram of $\mathbf{8 i .}$

Based on the literature reports, ${ }^{50}$ the mechanism involves the formation of an oxazolidinone intermediate. In the presence of activated (E)-3-benzylideneindolin-2-ones with $\mathrm{ZnBr}_{2}$, it undergoes loss of $\mathrm{CO}_{2}$ via a 

stereospecific 1,3-cycloreversion forming the azomethine ylide, which undergoes 1,3-dipolar cycloaddition to give the dispiro compounds $\mathbf{8 a - I}$ (Scheme 3).

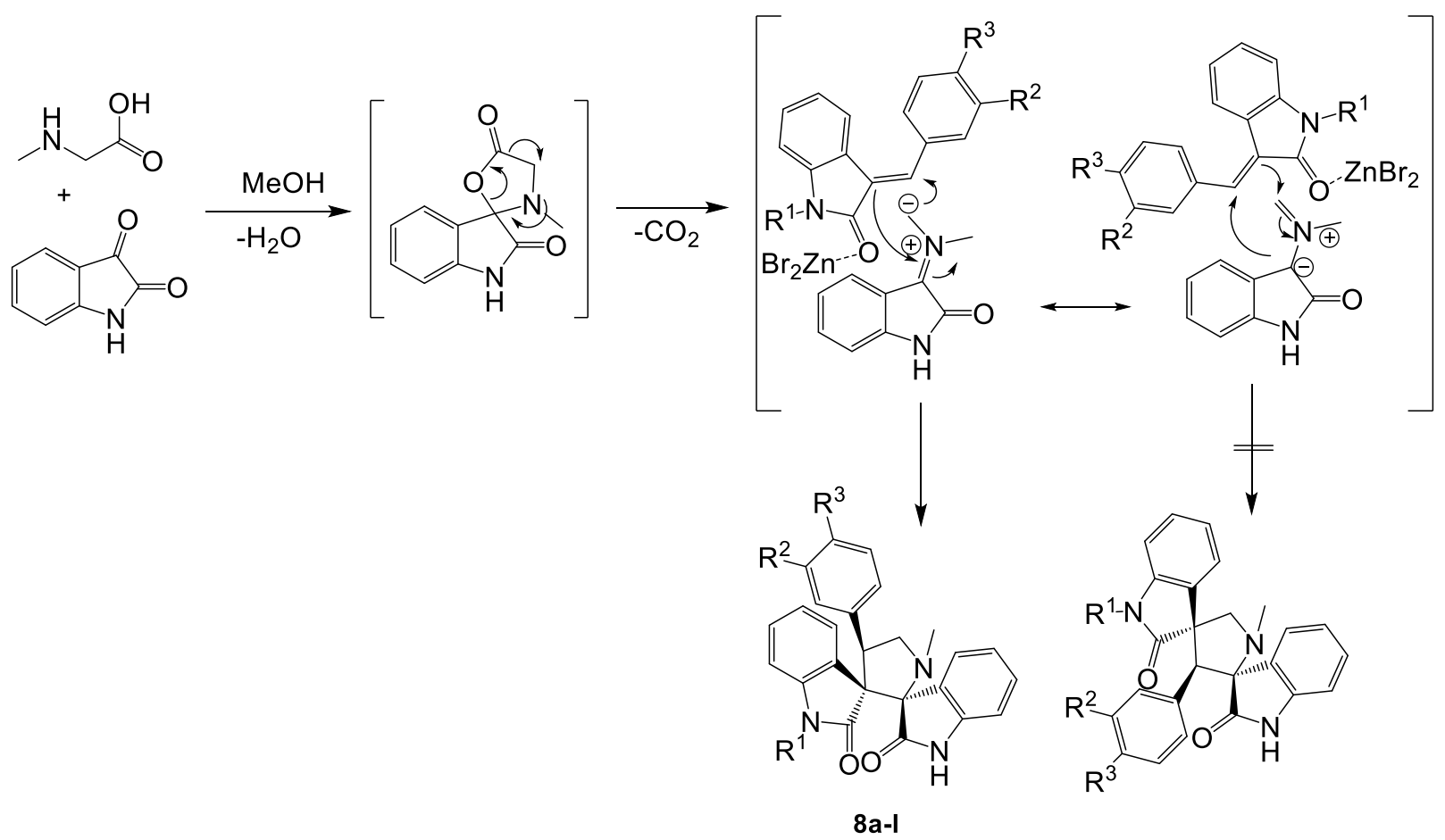

Scheme 3. Mechanism for the formation of compounds $8 \mathrm{a}-\mathbf{I}$.

\section{Conclusions}

This letter describes a facile, high yield and efficient method for the synthesis of novel dispiro compounds via 1,3-dipolar cycloaddition reaction of azomethine ylide generated from isatin and sarcosine with different (E)3-benzylideneindolin-2-ones by $\mathrm{ZnBr}_{2}$ catalyzed ultrasonic irradiation. The products were isolated by recrystallization in methanol without need for further purification. The synthetic route involves a regio- and stereo-controlled fashion as determined by NMR and confirmed by X-ray crystallographic analysis.

\section{Experimental Section}

General. The reagents and solvents were commercially available and purchased from Sigma-Aldrich and Merck, and were used without any additional purification. Ultrasonication was performed in a Parsonic 7500s ultrasonic bath with a frequency of $28 \mathrm{kHz}$ and a power of $100 \mathrm{~W}$. The liquid holding capacity of the ultrasonic cleaner tank was 6L. TLC: Silica-gel plates $60 \mathrm{~F}_{254}\left(\mathrm{SiO}_{2}\right.$; Merck). M.p.: Büchi melting point B-540 apparatus; in sealed capillaries. ${ }^{1} \mathrm{H}$ and ${ }^{13} \mathrm{C}$ NMR Spectra: Bruker (DRX-500 Avance) spectrometer at 500 and $300\left({ }^{1} \mathrm{H}\right)$ and 125 and $75.5\left({ }^{13} \mathrm{C}\right) \mathrm{MHz}$, in $\mathrm{CDCl}_{3}$ soln., at ambient temp.; $\delta$ in ppm rel. to $\mathrm{Me}_{4} \mathrm{Si}$ as internal standard, $J$ in $\mathrm{Hz}$. Signals of the ${ }^{13} \mathrm{C}$ NMR spectra corresponding to $\mathrm{CH}, \mathrm{CH}_{2}$, or $\mathrm{CH}_{3}$ groups are assigned from DEPT. Infrared spectra were recorded in an ATR apparatus. Mass spectrometric data (MS) were obtained by electron ionization (El, $70 \mathrm{eV}$ ), chemical ionization ( $\mathrm{Cl}$, isobutane) or electrospray ionization (ESI). 
General procedure for synthesis of the 3,3'-dispiropyrrolidine bisoxindole (8a-I). A mixture of (E)-3benzylideneindolin-2-one $7 \mathrm{a}-\mathrm{I}(1 \mathrm{mmol})$, isatin $(147 \mathrm{mg}, 1 \mathrm{mmol})$, sarcosine $(89 \mathrm{mg}, 1 \mathrm{mmol})$ and anhydrous $\mathrm{ZnBr}_{2}(20 \%, 45 \mathrm{mg}, 0.2 \mathrm{mmol})$ in methanol $(10 \mathrm{~mL})$ was sonicated for 30 minute at room temperature $(25-30$ $\left.{ }^{\circ} \mathrm{C}\right)$. After completion of the reaction as monitored by TLC, the mixture was poured in ice cold water and the precipitates were filtered and air dried. Then the product was recrystallized from methanol to afford the pure product $8 \mathrm{a}-\mathrm{I}$.

(3R*,3"S*,4'R*)-1',1"-Dimethyl-4'-phenyldispiro[indole-3,2'-pyrrolidine-3',3"-indole]-2,2" (1H,1"H)-dione

(8a). Pale yellow solid, yield 98\% (401 mg); mp 206-208 ${ }^{\circ} \mathrm{C}$. IR ( $\left.\mathrm{KBr}, \mathrm{v}, \mathrm{cm}^{-1}\right)$ : 3168 (m), 1698 (s), 1609 (s), 1468 (s). ${ }^{1} \mathrm{H}$ NMR $\left(500 \mathrm{MHz}, \mathrm{CDCl}_{3}\right): \delta 7.93(\mathrm{~s}, 1 \mathrm{H}, \mathrm{NH}), 7.53$ (d, J $\left.7.6 \mathrm{~Hz}, 1 \mathrm{H}, \mathrm{Ar}-\mathrm{H}\right), 7.26(\mathrm{~d}, J 7.4 \mathrm{~Hz}, 2 \mathrm{H}, \mathrm{Ar}-\mathrm{H}), 7.11-$ $7.14(\mathrm{~m}, 3 \mathrm{H}, \mathrm{Ar}-\mathrm{H}), 7.06(\mathrm{t}, J 7.2 \mathrm{~Hz}, 1 \mathrm{H}, \mathrm{Ar}-\mathrm{H}), 6.98(\mathrm{t}, J 7.6 \mathrm{~Hz}, 1 \mathrm{H}, \mathrm{Ar}-\mathrm{H}), 6.92(\mathrm{t}, J 7.6 \mathrm{~Hz}, 1 \mathrm{H}, \operatorname{Ar}-\mathrm{H}), 6.71(\mathrm{~d}, J$ $7.2 \mathrm{~Hz}, 1 \mathrm{H}, \mathrm{Ar}-\mathrm{H}), 6.55$ (d, J $7.6 \mathrm{~Hz}, 1 \mathrm{H}, \mathrm{Ar}-\mathrm{H}), 6.42(\mathrm{t}, J 7.1 \mathrm{~Hz}, 2 \mathrm{H}, \mathrm{Ar}-\mathrm{H}), 4.47-4.53\left(\mathrm{~m}, 2 \mathrm{H}, \mathrm{CH}_{2}\right), 3.73(\mathrm{t}, J 5.4$ $\mathrm{Hz}, 1 \mathrm{H}, \mathrm{CH}$ ), 2.96 (s, 3H, NMe), 2.30 (s, 3H, NMe). ${ }^{13} \mathrm{C} \mathrm{NMR}\left(125 \mathrm{MHz}, \mathrm{CDCl}_{3}\right): \delta 179.0(\mathrm{C}, \mathrm{N}-\mathrm{C}=\mathrm{O}), 177.5(\mathrm{C}, \mathrm{N}-$ $\mathrm{C}=\mathrm{O}), 144.2(\mathrm{C}), 142.0(\mathrm{C}), 138.4(\mathrm{C}), 130.0(\mathrm{CH}), 129.9(\mathrm{CH}), 128.5(\mathrm{CH}), 128.4(\mathrm{CH}), 128.3(\mathrm{CH}), 127.2(\mathrm{CH})$, $127.1(\mathrm{CH}), 125.5(\mathrm{C}), 125.0(\mathrm{C}), 122.8(\mathrm{CH}), 122.0(\mathrm{CH}), 109.7(\mathrm{CH}), 107.4(\mathrm{CH}), 79.5(\mathrm{C}), 64.9(\mathrm{C}), 57.2\left(\mathrm{CH}_{2}\right)$, $49.8\left(\mathrm{NCH}_{3}\right), 35.8(\mathrm{CH}), 26.6\left(\mathrm{NCH}_{3}\right) . \mathrm{MS}(\mathrm{GC}, 70 \mathrm{eV}): \mathrm{m} / \mathrm{z}(\%)=409\left(\mathrm{M}^{+}, 2\right), 235(31), 234(22), 174(100), 159$ (18). HRMS (ESI): calcd for $\mathrm{C}_{26} \mathrm{H}_{24} \mathrm{~N}_{3} \mathrm{O}_{2}(\mathrm{M}+\mathrm{H}) 410.1863$, found 410.1872.

$\left(3 R^{*}, 3^{\prime \prime} S^{*}, 4^{\prime} R^{*}\right)-4^{\prime}$-(4-Methoxyphenyl)-1',1"'-dimethyldispiro[indole-3,2' -pyrrolidine-3',3"'-indole]-

2,2"(1H,1"H)-dione (8b). Yellow solid, yield 90\% (396 mg); mp 216-218 ${ }^{\circ} \mathrm{C}$. IR (KBr, v, cm $\left.{ }^{-1}\right): 3165$ (m), 1702 (s), 1609 (s), 1493 (m). ${ }^{1} \mathrm{H}$ NMR (500 MHz, CDCl 3 ): $\delta 7.66$ (s, 1H, NH), 7.55 (d, J 7.5 Hz, 1H, Ar-H), 7.22 (d, J 8.6 $\mathrm{Hz}, 2 \mathrm{H}, \mathrm{Ar}-\mathrm{H}), 7.15(\mathrm{t}, J 7.5 \mathrm{~Hz}, 1 \mathrm{H}, \mathrm{Ar}-\mathrm{H}), 7.01(\mathrm{t}, J 7.6 \mathrm{~Hz}, 1 \mathrm{H}, \mathrm{Ar}-\mathrm{H}), 6.97(\mathrm{t}, J 7.8 \mathrm{~Hz}, 1 \mathrm{H}, \mathrm{Ar}-\mathrm{H}), 6.78(\mathrm{~d}, J 7.5 \mathrm{~Hz}$, $1 \mathrm{H}, \mathrm{Ar}-\mathrm{H}), 6.70(\mathrm{~d}, J 8.6 \mathrm{~Hz}, 2 \mathrm{H}, \mathrm{Ar}-\mathrm{H}), 6.56(\mathrm{~d}, J 7.6 \mathrm{~Hz}, 1 \mathrm{H}, \mathrm{Ar}-\mathrm{H}), 6.52(\mathrm{t}, J 7.6 \mathrm{~Hz}, 1 \mathrm{H}, \mathrm{Ar}-\mathrm{H}), 6.45(\mathrm{~d}, J 7.7 \mathrm{~Hz}$, $1 \mathrm{H}, \mathrm{Ar}-\mathrm{H}), 4.42-4.48\left(\mathrm{~m}, 2 \mathrm{H}, \mathrm{CH}_{2}\right), 3.72-3.80(\mathrm{~m}, 4 \mathrm{H}, \mathrm{OMe}, \mathrm{CH}), 3.00(\mathrm{~s}, 3 \mathrm{H}, \mathrm{NMe}), 2.35(\mathrm{~s}, 3 \mathrm{H}, \mathrm{NMe}) .{ }^{13} \mathrm{C} \mathrm{NMR}$ $\left(125 \mathrm{MHz}, \mathrm{CDCl}_{3}\right.$ ): $\delta 178.4$ (C, N-C=O), 162.8 (C, N-C=O), 158.6 (C), 143.3 (C), 142.1 (C), 130.8 (C), 130.4 (C), $129.8(\mathrm{CH}), 129.0(\mathrm{CH}), 128.3(\mathrm{CH}), 127.6(\mathrm{CH}), 125.6(\mathrm{CH}), 125.4(\mathrm{C}), 122.8(\mathrm{CH}), 121.8(\mathrm{CH}), 113.7(\mathrm{CH}), 109.6$ $(\mathrm{CH}), 107.4(\mathrm{CH}), 79.4(\mathrm{C}), 64.6(\mathrm{C}), 57.6\left(\mathrm{CH}_{2}\right), 55.5\left(\mathrm{OCH}_{3}\right), 49.2(\mathrm{CH}), 35.8\left(\mathrm{NCH}_{3}\right), 34.9\left(\mathrm{NCH}_{3}\right) . \mathrm{MS}(\mathrm{GC}, 70 \mathrm{eV})$ : $m / z(\%)=439\left(\mathrm{M}^{+}, 2\right), 277(5), 265$ (28), 264 (23), 174 (100), 159 (19). HRMS (ESI): calcd for $\mathrm{C}_{27} \mathrm{H}_{26} \mathrm{~N}_{3} \mathrm{O}_{3}(\mathrm{M}+\mathrm{H})$ 440.1968 , found 440.1973 .

$\left(3 R^{*}, 3^{\prime \prime} S^{*}, 4^{\prime} R^{*}\right)-4^{\prime}$-(4-Chlorophenyl)-1',1"-dimethyldispiro[indole-3,2'-pyrrolidine-3', 3"-indole]-

2,2"(1H,1"H)-dione (8c). White solid, yield 92\% (408 mg); mp 231-233 ${ }^{\circ} \mathrm{C} . \mathrm{IR}\left(\mathrm{KBr}, \mathrm{v}, \mathrm{cm}^{-1}\right): 3231(\mathrm{~m}), 1709(\mathrm{~s})$, 1695 (s), 1610 (m), 1369 (s). ${ }^{1} \mathrm{H}$ NMR (500 MHz, CDCl $): \delta 7.69$ (s, 1H, NH), 7.53 (d, J 7.6 Hz, 1H, Ar-H), $7.24(\mathrm{~d}, J$ 8.4 Hz ,2H, Ar-H), 7.13-7.17 (m, 3H, Ar-H), 6.98-7.03 (m, 2H, Ar-H), $6.71(\mathrm{~d}, J 7.5 \mathrm{~Hz}, 1 \mathrm{H}, \operatorname{Ar}-\mathrm{H}), 6.53-6.57(\mathrm{~m}$, $2 \mathrm{H}, \mathrm{Ar}-\mathrm{H}), 6.47(\mathrm{~d}, \mathrm{~J} 7.7 \mathrm{~Hz}, 1 \mathrm{H}, \mathrm{Ar}-\mathrm{H}), 4.42-4.47\left(\mathrm{~m}, 2 \mathrm{H}, \mathrm{CH}_{2}\right), 3.78(\mathrm{~m}, 1 \mathrm{H}, \mathrm{CH}), 3.01\left(\mathrm{~s}, 3 \mathrm{H}, \mathrm{NCH}_{3}\right), 2.34(\mathrm{~s}, 3 \mathrm{H}$, $\mathrm{NCH}_{3}$ ). ${ }^{13} \mathrm{C}$ NMR (125 MHz, $\mathrm{CDCl}_{3}$ ): $\delta 178.7$ (C, N-C=O), 177.3 (C, N-C=O), 144.2 (C), 141.9 (C), 136.9 (C), 132.9 (C), $131.4(\mathrm{CH}), 130.0(\mathrm{CH}), 128.7(\mathrm{CH}), 128.4(\mathrm{CH}), 127.6(\mathrm{CH}), 127.1(\mathrm{CH}), 125.3(\mathrm{C}), 124.6(\mathrm{C}), 122.9(\mathrm{CH})$, $122.2(\mathrm{CH}), 109.5(\mathrm{CH}), 107.4(\mathrm{CH}), 79.4(\mathrm{C}), 64.7(\mathrm{C}), 57.4\left(\mathrm{CH}_{2}\right), 49.1(\mathrm{CH}), 35.7\left(\mathrm{NCH}_{3}\right), 26.6\left(\mathrm{NCH}_{3}\right) . \mathrm{MS}(\mathrm{GC}$, 70eV): $m / z(\%)=443\left(\mathrm{M}^{+}, 1\right), 269(32), 268(14), 174(100), 159$ (17). HRMS (ESI): calcd for $\mathrm{C}_{26} \mathrm{H}_{23} \mathrm{CIN}_{3} \mathrm{O}_{2}(\mathrm{M}+\mathrm{H})$ 444.1473, found 444.1476.

(3R*,3"S*,4'R*)-1',1"'-Dimethyl-4' -(3-nitrophenyl)dispiro[indole-3,2'-pyrrolidine-3',3"'-indole]-2,2"$\left(\mathbf{H}, \mathbf{1}^{\prime \prime} \boldsymbol{H}\right)$-dione (8d). Bold yellow solid, yield 88\% (400 mg); mp 201-203 ${ }^{\circ} \mathrm{C}$. IR (KBr, v, $\left.\mathrm{cm}^{-1}\right): 3170(\mathrm{~m}), 1712$ (s), $1608(\mathrm{~m}), 1524$ (s). ${ }^{1} \mathrm{H}$ NMR $\left(500 \mathrm{MHz}, \mathrm{CDCl}_{3}\right): \delta 8.53(\mathrm{~s}, 1 \mathrm{H}, \mathrm{NH}), 7.37$ (d, J $\left.7.6 \mathrm{~Hz}, 1 \mathrm{H}, \operatorname{Ar}-\mathrm{H}\right), 7.01(\mathrm{t}, J 7.7$ $\mathrm{Hz}, 2 \mathrm{H}, \mathrm{Ar}-\mathrm{H}), 6.96(\mathrm{t}, J 7.6 \mathrm{~Hz}, 2 \mathrm{H}, \mathrm{Ar}-\mathrm{H}), 6.85(\mathrm{t}, J 7.6 \mathrm{~Hz}, 1 \mathrm{H}, \mathrm{Ar}-\mathrm{H}), 6.80(\mathrm{~d}, J 7.6 \mathrm{~Hz}, 1 \mathrm{H}, \mathrm{Ar}-\mathrm{H}), 6.54(\mathrm{t}, J 7.7 \mathrm{~Hz}$, $1 \mathrm{H}, \mathrm{Ar}-\mathrm{H}), 6.48(\mathrm{~d}, J 7.7 \mathrm{~Hz}, 1 \mathrm{H}, \mathrm{Ar}-\mathrm{H}), 6.45(\mathrm{~d}, J 7.6 \mathrm{~Hz}, 1 \mathrm{H}, \mathrm{Ar}-\mathrm{H}), 6.41(\mathrm{~d}, J 7.7 \mathrm{~Hz}, 1 \mathrm{H}, \mathrm{Ar}-\mathrm{H}), 6.17-6.18(\mathrm{~m}, 1 \mathrm{H}$, $\operatorname{Ar}-\mathrm{H}), 4.31(\mathrm{t}, J 7.4 \mathrm{~Hz}, 1 \mathrm{H}, \mathrm{CH}), 4.24\left(\mathrm{t}, J 8.7 \mathrm{~Hz}, 1 \mathrm{H}, \mathrm{CH}_{2}\right), 3.71(\mathrm{t}, J 8.2 \mathrm{~Hz}, 1 \mathrm{H}, \mathrm{CH}), 2.94\left(\mathrm{~s}, 3 \mathrm{H}, \mathrm{NCH}_{3}\right), 2.23(\mathrm{~s}$, $\left.3 \mathrm{H}, \mathrm{NCH}_{3}\right) .{ }^{13} \mathrm{C} \mathrm{NMR}\left(125 \mathrm{MHz}, \mathrm{CDCl}_{3}\right): \delta 178.3$ (C, N-C=O), 177.0 (C, N-C=O), 153.2 (C), 148.5 (C), 144.3 (C), $142.8(\mathrm{C}), 141.4(\mathrm{CH}), 139.7(\mathrm{CH}), 135.2(\mathrm{C}), 129.8(\mathrm{CH}), 128.6(\mathrm{CH}), 127.1(\mathrm{CH}), 126.8(\mathrm{CH}), 125.0(\mathrm{C}), 122.3$ 
$(\mathrm{CH}), 122.1(\mathrm{CH}), 110.3(\mathrm{CH}), 109.6(\mathrm{CH}), 108.7(\mathrm{CH}), 107.3(\mathrm{CH}), 79.1(\mathrm{C}), 62.7(\mathrm{C}), 56.8\left(\mathrm{CH}_{2}\right), 43.3\left(\mathrm{NCH}_{3}\right), 35.6$ (CH), $26.4\left(\mathrm{NCH}_{3}\right)$. MS (GC, 70eV): $\mathrm{m} / \mathrm{z}(\%)=454\left(\mathrm{M}^{+}, 2\right), 280$ (37), 174 (100), 159 (19). HRMS (ESI): calcd for $\mathrm{C}_{26} \mathrm{H}_{23} \mathrm{~N}_{4} \mathrm{O}_{4}(\mathrm{M}+\mathrm{H})$ 455.1713, found 455.1706 .

(3R*,3"S*,4'R*)-1"'-Ethyl-1'-methyl-4'-(3-nitrophenyl)dispiro[indole-3,2'-pyrrolidine-3',3"'-indole]-2,2"$\left(\mathbf{H}, \mathbf{1}^{\prime \prime} \boldsymbol{H}\right)$-dione (8e). Pale brown solid. yield 85\% (398 mg); mp 208-210 ${ }^{\circ} \mathrm{C} . \mathrm{IR}\left(\mathrm{KBr}, \mathrm{v}, \mathrm{cm}^{-1}\right): 3213(\mathrm{~m}), 1708$ (s), $1608(\mathrm{~m}), 1525$ (s). ${ }^{1} \mathrm{H}$ NMR (500 MHz, CDCl 3 ): $\delta 8.25$ (s, 1H, NH), $8.11(\mathrm{~s}, 1 \mathrm{H}, \mathrm{Ar}-\mathrm{H}), 7.93$ (d, J $6.9 \mathrm{~Hz}, 1 \mathrm{H}, \mathrm{Ar}-$ H), $7.54(\mathrm{~d}, J 7.6 \mathrm{~Hz}, 1 \mathrm{H}, \mathrm{Ar}-\mathrm{H}), 7.46(\mathrm{~d}, J 7.6 \mathrm{~Hz}, 1 \mathrm{H}, \mathrm{Ar}-\mathrm{H}), 7.27(\mathrm{t}, J 8.0 \mathrm{~Hz}, 1 \mathrm{H}, \mathrm{Ar}-\mathrm{H}), 7.13(\mathrm{t}, J 7.6 \mathrm{~Hz}, 1 \mathrm{H}, \mathrm{Ar}-$ H), $6.98(\mathrm{t}, J 7.6 \mathrm{~Hz}, 1 \mathrm{H}, \mathrm{Ar}-\mathrm{H}), 6.94(\mathrm{t}, J 7.7 \mathrm{~Hz}, 1 \mathrm{H}, \mathrm{Ar}-\mathrm{H}), 6.64(\mathrm{~d}, J 7.6 \mathrm{~Hz}, 1 \mathrm{H}, \mathrm{Ar}-\mathrm{H}), 6.59(\mathrm{~d}, J 7.7 \mathrm{~Hz}, 1 \mathrm{H}, \mathrm{Ar}-$ $\mathrm{H}), 6.48(\mathrm{~d}, J 7.8 \mathrm{~Hz}, 1 \mathrm{H}, \mathrm{Ar}-\mathrm{H}), 6.44(\mathrm{t}, J 7.5 \mathrm{~Hz}, 1 \mathrm{H}, \mathrm{Ar}-\mathrm{H}), 4.52\left(\mathrm{q}, J 7.1 \mathrm{~Hz}, 2 \mathrm{H}, \mathrm{NCH}_{2}\right), 3.78-3.82\left(\mathrm{~m}, 2 \mathrm{H}, \mathrm{CH}_{2}\right)$, 3.40-3.42 (m, 1H, CH), $2.33(\mathrm{~s}, 3 \mathrm{H}, \mathrm{NMe}), 0.94\left(\mathrm{t}, J 7.1 \mathrm{~Hz}, 3 \mathrm{H}, \mathrm{CH}_{3}\right) .{ }^{13} \mathrm{C} \mathrm{NMR}\left(125 \mathrm{MHz}, \mathrm{CDCl}_{3}\right): \delta 178.0(\mathrm{C}, \mathrm{N}-$ $\mathrm{C}=0$ ), 177.6 (C, N-C=O), 148.4 (C), 143.5 (C), 142.1 (C), $141.0(\mathrm{CH}), 136.3(\mathrm{CH}), 130.2(\mathrm{CH}), 129.1(\mathrm{CH}), 128.9$ $(\mathrm{CH}), 128.5(\mathrm{CH}), 127.6(\mathrm{CH}), 124.9(\mathrm{C}), 124.8(\mathrm{CH}), 124.4(\mathrm{C}), 123.1(\mathrm{CH}), 122.2(\mathrm{CH}), 122.0(\mathrm{CH}), 109.9(\mathrm{C})$, $108.0(\mathrm{CH}), 79.6(\mathrm{C}), 64.1(\mathrm{C}), 57.2\left(\mathrm{CH}_{2}\right), 49.7\left(\mathrm{CH}_{2}\right), 35.6\left(\mathrm{NCH}_{3}\right), 35.1(\mathrm{CH}), 12.8\left(\mathrm{CH}_{3}\right) . \mathrm{MS}(\mathrm{GC}, 70 \mathrm{eV}): \mathrm{m} / \mathrm{z}(\%)$ $=468\left(\mathrm{M}^{+}, 2\right), 294$ (21), 279 (13), 174 (100). HRMS (ESI): calcd for $\mathrm{C}_{27} \mathrm{H}_{25} \mathrm{~N}_{4} \mathrm{O}_{4}(\mathrm{M}+\mathrm{H})$ 469.1870, found 469.1878. $\left(3 R^{*}, 3^{\prime \prime} S^{*}, 4^{\prime} R^{*}\right)-1^{\prime \prime}$-Ethyl-1'-methyl-4' -(4-methylphenyl)dispiro[indole-3,2'-pyrrolidine-3', 3"'-indole]2,2"(1H,1"H)-dione (8f). White solid, yield 92\% (402 mg); mp 205-207 ${ }^{\circ} \mathrm{C}$. IR (KBr, v, cm $\left.{ }^{-1}\right): 3231(\mathrm{~m}), 1712(\mathrm{~s})$, 1699 (s), 1609 (s), 1467 (s). ${ }^{1} \mathrm{H}$ NMR (500 MHz, CDCl $): \delta 7.99$ (s, 1H, NH), 7.58 (d, J 7.5 Hz, 1H, Ar-H), 7.12-7.16 $(\mathrm{m}, 3 \mathrm{H}, \mathrm{Ar}-\mathrm{H}), 7.00-7.02(\mathrm{~m}, 1 \mathrm{H}, \mathrm{Ar}-\mathrm{H}), 6.93-6.96(\mathrm{~m}, 3 \mathrm{H}, \mathrm{Ar}-\mathrm{H}), 6.79(\mathrm{~d}, J 7.6 \mathrm{~Hz}, 1 \mathrm{H}, \mathrm{Ar}-\mathrm{H}), 6.59(\mathrm{~d}, J 7.6 \mathrm{~Hz}, 1 \mathrm{H}$, $\mathrm{Ar}-\mathrm{H}), 6.43-6.47(\mathrm{~m}, 2 \mathrm{H}, \mathrm{Ar}-\mathrm{H}), 4.48\left(\mathrm{~m}, 2 \mathrm{H}, \mathrm{NCH}_{2}\right), 3.70-3.83\left(\mathrm{~m}, 2 \mathrm{H}, \mathrm{CH}_{2}\right), 3.37-3.44(\mathrm{~m}, 1 \mathrm{H}, \mathrm{CH}), 2.34(\mathrm{~s}, 3 \mathrm{H}$, $\left.\mathrm{NCH}_{3}\right), 2.25\left(\mathrm{~s}, 3 \mathrm{H}, \mathrm{NCH}_{3}\right), 0.94\left(\mathrm{t}, J 7.2 \mathrm{~Hz}, 3 \mathrm{H}, \mathrm{CH}_{3}\right) .{ }^{13} \mathrm{C} \mathrm{NMR}\left(125 \mathrm{MHz}, \mathrm{CDCl}_{3}\right): \delta 178.3(\mathrm{C}, \mathrm{N}-\mathrm{C}=\mathrm{O}), 178.0(\mathrm{C}$, $\mathrm{N}-\mathrm{C}=\mathrm{O}), 143.3(\mathrm{C}), 142.1(\mathrm{C}), 136.3(\mathrm{C}), 135.2(\mathrm{C}), 129.7(\mathrm{CH}), 129.6(\mathrm{CH}), 129.1(\mathrm{CH}), 129.0(\mathrm{CH}), 128.3(\mathrm{CH})$, $127.7(\mathrm{CH}), 125.8(\mathrm{C}), 125.4(\mathrm{C}), 122.8(\mathrm{CH}), 121.7(\mathrm{CH}), 109.6(\mathrm{CH}), 107.3(\mathrm{CH}), 79.5(\mathrm{C}), 64.6(\mathrm{C}), 57.3\left(\mathrm{CH}_{2}\right)$, $49.7(\mathrm{CH}), 35.8\left(\mathrm{NCH}_{3}\right), 34.9\left(\mathrm{CH}_{2}\right), 21.4\left(\mathrm{CH}_{3}\right), 12.8\left(\mathrm{CH}_{3}\right) . \mathrm{MS}(\mathrm{GC}, 70 \mathrm{eV}): \mathrm{m} / \mathrm{z}(\%)=437\left(\mathrm{M}^{+}, 3\right), 291(8), 263$ (30), 174 (100). HRMS (ESI): calcd for $\mathrm{C}_{28} \mathrm{H}_{28} \mathrm{~N}_{3} \mathrm{O}_{2}(\mathrm{M}+\mathrm{H})$ 438.2176, found 438.2182.

(3R*,3" $\left.\mathrm{S}^{*}, 4^{\prime} \mathrm{R}^{*}\right)-4^{\prime}$-(4-Chlorophenyl)-1"'-ethyl-1'-methyldispiro[indole-3,2' -pyrrolidine-3',3"'-indole]2,2"(1H,1"H)-dione (8g). White solid, yield 95\% (435 mg); mp 209-211 ${ }^{\circ} \mathrm{C}$. IR (KBr, v, cm-1): $3274(\mathrm{~m}), 1709$ (s), 1682 (s), 1606 (m). ${ }^{1} \mathrm{H}$ NMR (500 MHz, CDCl 3 ): $\delta 7.57$ (s, 1H, NH), 7.44 (d, J $\left.7.6 \mathrm{~Hz}, 1 \mathrm{H}, \mathrm{Ar}-\mathrm{H}\right), 7.19$ (d, J $8.4 \mathrm{~Hz}$, $2 \mathrm{H}, \mathrm{Ar}-\mathrm{H}), 7.08(\mathrm{~d}, J 8.4 \mathrm{~Hz}, 2 \mathrm{H}, \mathrm{Ar}-\mathrm{H}), 7.05(\mathrm{t}, J 7.6 \mathrm{~Hz}, 1 \mathrm{H}, \mathrm{Ar}-\mathrm{H}), 6.97(\mathrm{t}, J 7.6 \mathrm{~Hz}, 1 \mathrm{H}, \mathrm{Ar}-\mathrm{H}), 6.88(\mathrm{t}, J 7.6 \mathrm{~Hz}$, $1 \mathrm{H}, \operatorname{Ar}-\mathrm{H}), 6.80(\mathrm{~d}, J 7.6 \mathrm{~Hz}, 1 \mathrm{H}, \mathrm{Ar}-\mathrm{H}), 6.55(\mathrm{~d}, J 7.7 \mathrm{~Hz}, 1 \mathrm{H}, \mathrm{Ar}-\mathrm{H}), 6.52(\mathrm{t}, J 7.7 \mathrm{~Hz}, 1 \mathrm{H}, \mathrm{Ar}-\mathrm{H}), 6.47(\mathrm{~d}, J 7.7 \mathrm{~Hz}$, $1 \mathrm{H}, \operatorname{Ar}-\mathrm{H}), 4.46(\mathrm{t}, J 8.5 \mathrm{~Hz}, 1 \mathrm{H}, \mathrm{CH}), 4.37\left(\mathrm{t}, J 8.0 \mathrm{~Hz}, 1 \mathrm{H}, \mathrm{CH}_{2}\right), 3.72-3.79\left(\mathrm{~m}, 1 \mathrm{H}, \mathrm{NCH}_{2}\right), 3.66(\mathrm{t}, J 8.0 \mathrm{~Hz}, 1 \mathrm{H}$, $\mathrm{CH}_{2}$ ), 3.34-3.41 (m, $\left.1 \mathrm{H}, \mathrm{NCH}_{2}\right), 2.29(\mathrm{~s}, 3 \mathrm{H}, \mathrm{NMe}), 0.90\left(\mathrm{t}, J 7.2 \mathrm{~Hz}, 3 \mathrm{H}, \mathrm{CH}_{3}\right) .{ }^{13} \mathrm{C} \mathrm{NMR}\left(125 \mathrm{MHz}, \mathrm{CDCl}_{3}\right): \delta 183.1$ (C, N-C=O), 182.0 (C, N-C=O), 148.2 (C), 144.2 (C), 142.1 (C), 137.2 (C), 137.1(CH), 136.0 (C), 134.5 (CH), 133.7 $(\mathrm{CH}), 133.3(\mathrm{CH}), 133.0(\mathrm{CH}), 131.8(\mathrm{CH}), 130.0(\mathrm{C}), 126.8(\mathrm{CH}), 126.3(\mathrm{CH}), 114.6(\mathrm{CH}), 112.3(\mathrm{CH}), 83.9(\mathrm{C}), 68.9$ (C), $62.1\left(\mathrm{CH}_{2}\right), 54.3\left(\mathrm{NCH}_{3}\right), 40.4(\mathrm{CH}), 39.6\left(\mathrm{CH}_{2}\right), 17.5\left(\mathrm{CH}_{3}\right) . \mathrm{MS}(\mathrm{GC}, 70 \mathrm{eV}): \mathrm{m} / z(\%)=457\left(\mathrm{M}^{+}, 3\right), 291(16)$, 283 (15), 175 (14), 174 (100), 159 (12). HRMS (ESI): calcd for $\mathrm{C}_{27} \mathrm{H}_{25} \mathrm{CIN}_{3} \mathrm{O}_{2}$ (M+H) 458.1629, found 458.1639.

(3R*,3"S*,4'R*)-1"-Ethyl-4'-(4-hydroxyphenyl)-1'-methyldispiro[indole-3,2'-pyrrolidine-3',3"indole]-2,2" -

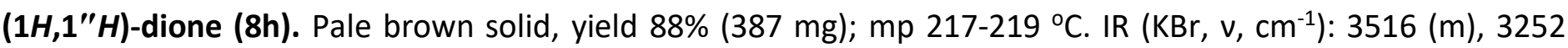
(w), 1697 (s), 1681 (s), 1610 (s), 1467 (s). ${ }^{1} \mathrm{H}$ NMR (500 MHz, CDCl $): \delta 9.10$ (s, 1H, OH), 7.84 (s, 1H, NH), 7.52 (d, J $7.5 \mathrm{~Hz}, 1 \mathrm{H}, \mathrm{Ar}-\mathrm{H}), 7.31$ (d, J $8.0 \mathrm{~Hz}, 1 \mathrm{H}, \mathrm{Ar}-\mathrm{H}), 6.86$ (t, J $7.6 \mathrm{~Hz}, 1 \mathrm{H}, \mathrm{Ar}-\mathrm{H}), 6.69-6.73(\mathrm{~m}, 3 \mathrm{H}, \mathrm{Ar}-\mathrm{H}), 6.63(\mathrm{t}, J$ $7.4 \mathrm{~Hz}, 1 \mathrm{H}, \mathrm{Ar}-\mathrm{H}), 6.39$ (d, J $7.8 \mathrm{~Hz}, 1 \mathrm{H}, \mathrm{Ar}-\mathrm{H}), 6.31(\mathrm{~d}, J 7.6 \mathrm{~Hz}, 1 \mathrm{H}, \mathrm{Ar}-\mathrm{H}), 6.20-6.24(\mathrm{~m}, 3 \mathrm{H}, \mathrm{Ar}-\mathrm{H}), 4.40(\mathrm{~m}, 2 \mathrm{H}$, $\left.\mathrm{CH}_{2}\right), 3.43-3.52\left(\mathrm{~m}, 3 \mathrm{H}, \mathrm{CH}_{2}, \mathrm{CH}\right), 2.12(\mathrm{~s}, 3 \mathrm{H}, \mathrm{NMe}), 0.73\left(\mathrm{t}, J 7.1 \mathrm{~Hz}, 3 \mathrm{H}, \mathrm{CH}_{3}\right) .{ }^{13} \mathrm{C} \mathrm{NMR}\left(125 \mathrm{MHz}, \mathrm{CDCl}_{3}\right): \delta$ 178.9 (C, N-C=O), 177.3 (C, N-C=O), 156.0 (C), 144.0 (C), 143.3 (C), 129.3 (CH), $128.4(\mathrm{CH}), 127.5$ (CH), 127.3 $(\mathrm{CH}), 127.0(\mathrm{CH}), 126.4(\mathrm{C}), 125.6(\mathrm{C}), 125.5(\mathrm{C}), 120.5(\mathrm{CH}), 118.8(\mathrm{CH}), 114.5(\mathrm{CH}), 109.4(\mathrm{CH}), 106.5(\mathrm{CH}), 79.0$ (C), $63.5(\mathrm{C}), 57.7\left(\mathrm{CH}_{2}\right), 42.8(\mathrm{CH}), 35.7\left(\mathrm{NCH}_{3}\right), 34.4\left(\mathrm{CH}_{2}\right), 12.5\left(\mathrm{CH}_{3}\right) . \mathrm{MS}(\mathrm{GC}, 70 \mathrm{eV}): \mathrm{m} / \mathrm{z}(\%)=439\left(\mathrm{M}^{+}, 6\right)$, 368 (9), 174 (100), 159 (11), 111 (15). HRMS (ESI): calcd for $\mathrm{C}_{27} \mathrm{H}_{26} \mathrm{~N}_{3} \mathrm{O}_{3}(\mathrm{M}+\mathrm{H})$ 440.1968, found 440.1968. 
(3R*,3"S* ,4'R*)-4' -(4-Bromophenyl)-1"'-ethyl-1'-methyldispiro[indole-3,2'-pyrrolidine-3',3"'-indole]-2,2"-

$\left(\mathbf{H} \mathbf{H} \mathbf{1}^{\prime \prime} \boldsymbol{H}\right)$-dione (8i). White solid, yield 97\% (487 mg); mp 216-218 ${ }^{\circ} \mathrm{C} . \mathrm{IR}\left(\mathrm{KBr}, \mathrm{v}, \mathrm{cm}^{-1}\right)$ : 3269 (m), 1709 (s), 1681 (s), $1606(\mathrm{~m}) .{ }^{1} \mathrm{H}$ NMR (300 MHz, CDCl 3 ): $\delta 7.49$ (d, J $\left.7.4 \mathrm{~Hz}, 1 \mathrm{H}, \mathrm{Ar}-\mathrm{H}\right), 7.26(\mathrm{~s}, 1 \mathrm{H}, \mathrm{NH}), 7.16-7.20(\mathrm{~m}, 2 \mathrm{H}, \mathrm{Ar}-\mathrm{H})$, 7.02-7.08 (m, 3H, Ar-H), 6.91 (tt, J $7.7 \mathrm{~Hz}, 1.4 \mathrm{~Hz}, 2 \mathrm{H}, \mathrm{Ar}-\mathrm{H}), 6.69$ (dd, 1H, J 7.7 Hz, 0.7 Hz, Ar-H), 6.39-6.50 (m, $3 \mathrm{H}, \mathrm{Ar}-\mathrm{H}), 4.32-4.36\left(\mathrm{~m}, 2 \mathrm{H}, \mathrm{CH}_{2}\right), 3.61-3.77\left(\mathrm{~m}, 2 \mathrm{H}, \mathrm{NCH}_{2}, \mathrm{CH}\right), 3.24-3.36\left(\mathrm{~m}, 1 \mathrm{H}, \mathrm{NCH}_{2}\right), 2.25\left(\mathrm{~s}, 3 \mathrm{H}, \mathrm{NCH}_{3}\right)$, $0.83\left(\mathrm{t}, \mathrm{J} 7.3 \mathrm{~Hz}, 3 \mathrm{H}, \mathrm{CH}_{3}\right) .{ }^{13} \mathrm{C}$ NMR (75.5 MHz, $\mathrm{CDCl}_{3}$ ): $\delta 177.8$ (C, N-C=O), 176.9 (C, N-C=O), 143.0 (C), 141.4 (C), $137.1(\mathrm{C}), 131.2(\mathrm{CH}), 131.0(\mathrm{CH}), 129.5(\mathrm{CH}), 128.4(\mathrm{CH}), 128.2(\mathrm{CH}), 127.2(\mathrm{CH}), 125.0(\mathrm{C}), 124.5(\mathrm{C}), 122.6$ $(\mathrm{CH}), 121.5(\mathrm{CH}), 120.6(\mathrm{C}), 109.2(\mathrm{CH}), 107.3(\mathrm{CH}), 78.9(\mathrm{C}), 64.0(\mathrm{C}), 56.9\left(\mathrm{CH}_{2}\right), 49.1(\mathrm{CH}), 35.3\left(\mathrm{NCH}_{3}\right), 34.6$ $\left(\mathrm{CH}_{2}\right), 12.4\left(\mathrm{CH}_{3}\right)$. MS (GC, 70eV): $\mathrm{m} / z(\%)=501\left(\mathrm{M}^{+}, 1\right), 329$ (27), 327 (27), 174 (100), 159 (24). HRMS (ESI): calcd for $\mathrm{C}_{27} \mathrm{H}_{25} \mathrm{BrN}_{3} \mathrm{O}_{2}(\mathrm{M}+\mathrm{H}) 502.1130$, found 502.1133 .

(3R*,3"' $\left.{ }^{*}, 4^{\prime} R^{*}\right)-1^{\prime}-M e t h y l-4 '-(4-m e t h y l p h e n y l)-1^{\prime \prime}$-phenyldispiro[indole-3,2'-pyrrolidine-3',3"'-indole]-2,2"-

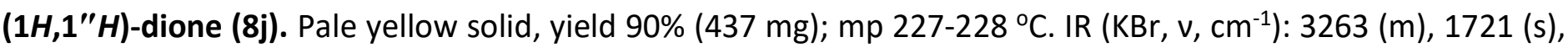
1703 (s). ${ }^{1} \mathrm{H}$ NMR (500 MHz, CDCl 3 ): $\delta 8.11$ (s, 1H, NH), 7.52 (d, J $\left.7.5 \mathrm{~Hz}, 1 \mathrm{H}, \mathrm{Ar}-\mathrm{H}\right), 7.40$ (t, J 7.5 Hz, 2H, Ar-H), $7.34(\mathrm{t}, J 7.3 \mathrm{~Hz}, 1 \mathrm{H}, \mathrm{Ar}-\mathrm{H})$, 7.15-7.22 (m, 3H, Ar-H), 6.94-7.02 (m, 5H, Ar-H), 6.79-6.82 (m, 2H, Ar-H), 6.59 (d, J $7.6 \mathrm{~Hz}, 1 \mathrm{H}, \mathrm{Ar}-\mathrm{H}), 6.45-6.48(\mathrm{~m}, 1 \mathrm{H}, \mathrm{Ar}-\mathrm{H}), 6.28(\mathrm{~d}, \mathrm{~J} 8.0 \mathrm{~Hz}, 1 \mathrm{H}, \mathrm{Ar}-\mathrm{H}), 4.53-4.60(\mathrm{~m}, 1 \mathrm{H}, \mathrm{CH}), 3.71-3.74(\mathrm{~m}, 2 \mathrm{H}$, $\mathrm{CH}_{2}$ ), 2.34 (s, 3H, NMe), 2.24 (s, 3H, Me). ${ }^{13} \mathrm{C} \mathrm{NMR} \mathrm{(125} \mathrm{MHz,} \mathrm{CDCl} 3$ ): $\delta 181.5$ (C, N-C=O), 178.1 (C, N-C=O), 144.5 (C), 142.3 (C), 136.5 (C), 135.0 (C), 134.8 (C), $129.9(\mathrm{CH}), 129.8(\mathrm{CH}), 129.7(\mathrm{CH}), 129.1(\mathrm{CH}), 129.0(\mathrm{CH})$, $128.5(\mathrm{CH}), 128.3(\mathrm{CH}), 127.8(\mathrm{CH}), 127.6(\mathrm{CH}), 125.5(\mathrm{C}), 124.9(\mathrm{C}), 122.9(\mathrm{CH}), 122.2(\mathrm{CH}), 109.7(\mathrm{CH}), 108.6$ $(\mathrm{CH}), 79.8(\mathrm{C}), 65.1(\mathrm{C}), 57.3\left(\mathrm{CH}_{2}\right), 49.8\left(\mathrm{NCH}_{3}\right), 35.8(\mathrm{CH}), 21.5\left(\mathrm{CH}_{3}\right) . \mathrm{MS}(\mathrm{GC}, 70 \mathrm{eV}): \mathrm{m} / \mathrm{z}(\%)=485\left(\mathrm{M}^{+}, 1\right)$, 312 (22), 311 (100), 310 (51), 174 (90). HRMS (ESI): calcd for $\mathrm{C}_{32} \mathrm{H}_{28} \mathrm{~N}_{3} \mathrm{O}_{2}(\mathrm{M}+\mathrm{H})$ 486.217, found 486.2180.

(3R*,3"S*,4'R*)-4'-(4-Methoxyphenyl)-1'-methyl-1"'-phenyldispiro[indole-3,2'-pyrrolidine-3',3"'-indole]2,2"(1H,1"H)-dione (8k). Bold yellow solid, yield 86\% (431 mg); mp 215-217 ${ }^{\circ} \mathrm{C}$. IR (KBr, v, cm $\left.{ }^{-1}\right): 3235(\mathrm{~m})$, 1712 (s), 1696 (s), 1609 (m), 1511 (m). ${ }^{1} \mathrm{H}$ NMR (500 MHz, CDCl $): \delta 7.64$ (s, 1H, Ar-H), 7.56 (d, J 7.5 Hz, 1H, Ar$\mathrm{H}), 7.45(\mathrm{t}, J 7.3 \mathrm{~Hz}, 2 \mathrm{H}, \mathrm{Ar}-\mathrm{H}), 7.37-7.40(\mathrm{~m}, 1 \mathrm{H}, \mathrm{Ar}-\mathrm{H}), 7.27-7.30(\mathrm{~m}, 2 \mathrm{H}, \mathrm{Ar}-\mathrm{H}), 7.21(\mathrm{t}, J 7.5 \mathrm{~Hz}, 1 \mathrm{H}, \mathrm{Ar}-\mathrm{H})$, 7.00-7.04 (m, 3H, Ar-H), 6.85-6.90 (m, 2H, Ar-H), $6.75(\mathrm{~d}, J 8.6 \mathrm{~Hz}, 2 \mathrm{H}, \mathrm{Ar}-\mathrm{H}), 6.63(\mathrm{~d}, J 7.6 \mathrm{~Hz}, 1 \mathrm{H}, \mathrm{Ar}-\mathrm{H}), 6.56(\mathrm{t}$, J $7.6 \mathrm{~Hz}, 1 \mathrm{H}, \mathrm{Ar}-\mathrm{H}), 6.34\left(\mathrm{~d}, J 7.7 \mathrm{~Hz}, 1 \mathrm{H}, \mathrm{Ar}-\mathrm{H}\right.$ ), 4.60 (dd, J 9.2, 8.7 Hz, $1 \mathrm{H}, \mathrm{CH}_{2}$ ), 4.52 (dd, J 9.3, 9.2 Hz, $1 \mathrm{H}, \mathrm{CH}_{2}$ ), 3.77-3.80 (m, 4H, OMe, CH), $2.38\left(\mathrm{~s}, 3 \mathrm{H}, \mathrm{NCH}_{3}\right) .{ }^{13} \mathrm{C} \mathrm{NMR}\left(125 \mathrm{MHz}, \mathrm{CDCl}_{3}\right): \delta 178.0(\mathrm{C}, \mathrm{N}-\mathrm{C}=\mathrm{O}), 177.1$ (C, N$\mathrm{C}=0), 158.7$ (C), 144.4 (C), 142.0 (C), 134.9 (C), 130.9 (CH), 130.2 (C), 129.9 (CH), 129.7 (CH), 129.0 (CH), 128.4 $(\mathrm{CH}), 128.3(\mathrm{CH}), 127.9(\mathrm{CH}), 127.6(\mathrm{CH}), 125.6(\mathrm{C}), 124.9(\mathrm{C}), 123.0(\mathrm{CH}), 122.3(\mathrm{CH}), 113.8(\mathrm{CH}), 109.6(\mathrm{CH})$, $108.6(\mathrm{CH}), 79.8(\mathrm{C}), 65.1(\mathrm{C}), 57.6\left(\mathrm{CH}_{2}\right), 55.4\left(\mathrm{OCH}_{3}\right), 49.5(\mathrm{CH}), 35.8\left(\mathrm{NCH}_{3}\right) . \mathrm{MS}(\mathrm{GC}, 70 \mathrm{eV}): \mathrm{m} / \mathrm{z}(\%)=501$ $\left(\mathrm{M}^{+}, 2\right), 328$ (47), 327 (100), 326 (57), 284 (29), 174 (99). HRMS (ESI): calcd for $\mathrm{C}_{32} \mathrm{H}_{28} \mathrm{~N}_{3} \mathrm{O}_{3}(\mathrm{M}+\mathrm{H})$ 502.2125, found 502.2121.

$\left(3 R^{*}, 3^{\prime \prime} S^{*}, 4^{\prime} R^{*}\right)-4^{\prime}$-(4-Chlorophenyl)-1'-methyl-1"'-phenyldispiro[indole-3,2'-pyrrolidine-3',3"'-indole]2,2"(1H,1"H)-dione (8I). Pale gray solid, yield 94\% (476 mg); mp 214-216 ${ }^{\circ} \mathrm{C} . \mathrm{IR}\left(\mathrm{KBr}, \mathrm{v}, \mathrm{cm}^{-1}\right): 3245(\mathrm{~m}), 1708$ (s), 1688 (s), 1608 (m), $1493(\mathrm{~m}) .{ }^{1} \mathrm{H}$ NMR $\left(500 \mathrm{MHz}, \mathrm{CDCl}_{3}\right): \delta 8.12$ (s, 1H, NH), 7.42 (d, J $\left.7.4 \mathrm{~Hz}, 1 \mathrm{H}, \mathrm{Ar}-\mathrm{H}\right), 7.33$ (t, J 7.3 Hz, 2H, Ar-H), 7.28 (d, J 7.3 Hz, 1H, Ar-H), 7.19 (d, J 7.8 Hz, 2H, Ar-H), 7.07-7.12 (m, 3H, Ar-H), 6.87-6.92 $(\mathrm{m}, 3 \mathrm{H}, \mathrm{Ar}-\mathrm{H}), 6.77(\mathrm{t}, J 7.4 \mathrm{~Hz}, 1 \mathrm{H}, \mathrm{Ar}-\mathrm{H}), 6.68(\mathrm{~d}, J 7.2 \mathrm{~Hz}, 1 \mathrm{H}, \mathrm{Ar}-\mathrm{H}), 6.51(\mathrm{~d}, J 7.7 \mathrm{~Hz}, 1 \mathrm{H}, \mathrm{Ar}-\mathrm{H}), 6.39(\mathrm{t}, J 7.6$ $\mathrm{Hz}, 1 \mathrm{H}, \mathrm{Ar}-\mathrm{H}), 6.23$ (d, J 7.8 Hz, 1H, Ar-H), 4.40-4.48 (m, 2H, CH 2$), 3.60-3.67(\mathrm{~m}, 1 \mathrm{H}, \mathrm{CH}), 2.25(\mathrm{~s}, 3 \mathrm{H}, \mathrm{NMe}) .{ }^{13} \mathrm{C}$ NMR (125 MHz, CDCl $): \delta 182.0$ (C, N-C=O), 178.0 (C, N-C=O), 144.5 (C), 142.3(C), 136.9 (C), 134.7 (C), 133.0 (C), $131.3(\mathrm{CH}), 130.1(\mathrm{CH}), 129.8(\mathrm{CH}), 128.8(\mathrm{CH}), 128.7(\mathrm{CH}), 128.6(\mathrm{CH}), 128.5(\mathrm{CH}), 127.7(\mathrm{CH}), 127.5(\mathrm{CH})$, $125.3(\mathrm{C}), 124.5(\mathrm{C}), 123.0(\mathrm{CH}), 122.4(\mathrm{CH}), 109.8(\mathrm{CH}), 108.8(\mathrm{CH}), 79.8(\mathrm{C}), 64.9(\mathrm{C}), 57.3\left(\mathrm{CH}_{2}\right), 49.5(\mathrm{CH})$, 35.7 $\left(\mathrm{NCH}_{3}\right)$. MS (GC, 70eV): $m / z(\%)=505\left(\mathrm{M}^{+}, 1\right), 331$ (79), 267 (35), 220 (46), 175 (32), 174 (100). HRMS (ESI): calcd for $\mathrm{C}_{31} \mathrm{H}_{25} \mathrm{~N}_{3} \mathrm{O}_{2}(\mathrm{M}+\mathrm{H})$ 506.1629, found 506.1627 . 


\section{Acknowledgements}

We gratefully acknowledge financial support from the Research Council of University of Qom.

\section{References}

1. Pandey, G.; Banerjee, P.; Gadre, S. R. Chem. Rev. 2006, 106, 4484. http://dx.doi.org/10.1021/cr050011g

2. Coldham, I.; Hufton, R. Chem. Rev. 2005, 105, 2765. http://dx.doi.org/10.1021/cr040004c

3. Gothelf, K. V.; Jorgensen, K. A. Chem. Rev. 1998, 98, 863. http://dx.doi.org/10.1021/cr970324e

4. Lashgari, N.; Ziarani, G. M. Arkivoc 2012, (i), 277. http://dx.doi.org/10.3998/ark.5550190.0013.108

5. Rajesh, R.; Raghunathan R. Tetrahedron Lett. 2010, 51, 5845. http://dx.doi.org/10.1016/i.tetlet.2010.09.002

6. Ghandi, M.; Taheri, A.; Abbasi A. Tetrahedron 2010, 66, 6744. http://dx.doi.org/10.1016/i.tet.2010.06.078

7. Lakshmi, N. V.; Thirumurugan, P.; Jayakumar, C.; Perumal P. T. Synlett 2010, 6, 955.

8. Bergman, J. Adv. Heterocycl. Chem. 2015, 117, 1. http://dx.doi.org/10.1016/bs.aihch.2015.08.001

9. Edmondson, S. D.; Danishefsky, S. J. Angew. Chem. 1998, 110, 1190. http://dx.doi.org/10.1002/(SICI)1521-375719980420110:8<1190::AID-ANGE1190>3.0.CO;2-S

10. Hussein, E. M.; Abdel-Monem, M. I. Arkivoc 2011, (x), 85. http://dx.doi.org/10.3998/ark.5550190.0012.a07

11. Galliford, C. V.; Scheidt, K. A. Angew. Chem., Int. Ed. 2007, 46, 8748. http://dx.doi.org/10.1002/anie.200701342

12. Trost, B.; Brennan, M. K. Synthesis 2009, 3003.

13. Millemaggi, A.; Taylor, R. J. K. Eur. J. Org. Chem. 2010, 4527. http://dx.doi.org/10.1002/ejoc.201090064

14. Colegate, S. M.; Anderton, N.; Edgar, J.; Bourke, C. A.; Oram, R. N. Aust. Vet. J. 1999, $77,537$.

15. Kornet, M. J.; Thio, A. P. J. Med. Chem. 1976, 19, 892. http://dx.doi.org/10.1021/jm00229a007

16. Ding, K.; Lu, Y.; Nikolovska-Coleska, Z.; Wang, G.; Qiu, S.; Shangary, S.; Gao, W.; Qin, D.; Stuckey, J.; Krajewski, K.; Roller, P. P.; Wang, S. J. Med. Chem. 2006, 49, 3432. http://dx.doi.org/10.1021/im051122a

17. Yu, S.; Qin, D.; Shangary, S.; Chen, J.; Wang, G.; Ding, K.; McEachern, D.; Qiu, S.; Nikolovska-Coleska, Z.; Miller, R.; Kang, S.; Yang, D.; Wang, S. J. Med. Chem. 2009, 52, 7970.

http://dx.doi.org/10.1021/im901400z

18. Garnick, R. L.; LeQuesne, P. W. J. Am. Chem. Soc. 1978, 100, 4213. http://dx.doi.org/10.1021/ja00481a034

19. Jossang, A.; Jossang, P.; Hadi, H. A.; Sevenet, T.; Bodo, B. J. Org. Chem. 1991, 56, 6527. http://dx.doi.org/10.1021/jo00023a016 
20. Dideberg, O.; Lamotte-Brasseur, J.; Dupont, L.; Campsteyn, H.; Vermeire, M.; Angenot, L.; Acta Crystallogr. Sect. B 1977, 33, 1796. http://dx.doi.org/10.1107/S0567740877007080

21. Abou-Gharbia, M. A.; Doukas, P. H. Heterocycles 1979, 12, 637.

22. Ziarani, G. M.; Moradi, R.; Lashgari, N. Arkivoc 2016, (i), 1. http://dx.doi.org/10.3998/ark.5550190.p009.385

23. Liu, J.; Sun, H.; Liu, X.; Ouyang, L.; Kang, T.; Xie, Y.; Wang, X. Tetrahedron Lett. 2012, 53, 2336. http://dx.doi:10.1016/j.tetlet.2012.02.099

24. Velikorodov, A. V.; Poddubnyi, O. Yu.; Krivosheev, O. O.; Titova, O. L. Russ. J. Org. Chem. 2011, 47, 402. http://dx.doi:10.1134/S1070428011030122

25. Lakshmi, N. V.; Thirumurugan, P.; Perumal P. T. Tetrahedron Lett. 2010, 51, 1064. http://dx.doi:10.1016/j.tetlet.2009.12.079

26. Kandrthikeyan, K.; Saranya, N.; Kalaivani, A.; Perumal P. T. Synlett 2010, 18, 2751.

27. Babu, A. R. S.; Raghunathan, R. Tetrahedron Lett. 2007, 48, 305. http://dx.doi:10.1016/j.tetlet.2006.11.012

28. Shanmugam, P.; Viswambharan, B.; Madhavan, S. Org. Lett. 2007, 9, 4095. http://dx.doi:10.1021/ol701533d

29. Shanmugam, P.; Viswambharan, B.; Selvakumar, K.; Madhavan, S. Tetrahedron Lett. 2008, 49, 2611. http://dx.doi:10.1016/j.tetlet.2008.02.104

30. Muthusamy, S.; Kumar, S. G. Tetrahedron 2016, 72, 2392. http://dx.doi.org/10.1016/j.tet.2016.03.046

31. Liu, Y.-Y.; Duan, S.-W.; Zhang, R.; Liu, Y.-H.; Chen, J.-R.; Xiao, W.-J. Org. Biomol. Chem. 2016, $14,5224$. http://dx.doi. 10.1039/C6OB00891G

32. Ziarani, G. M.; Moradi, R.; Lashgari, N. Tetrahedron: Asymmetry 2015, 26, 517. http://dx.doi.org/10.1016/j.tetasy.2015.04.011

33. Babu, A. R. S.; Raghunathan, R. Tetrahedron Lett. 2007, 48, 6809. http://dx.doi:10.1016/i.tetlet.2007.07.085

34. Habibi, D.; Nasrollahzadeh, M.; Sahebekhtiari H.; Parish, R. V. Tetrahedron 2013, 69, 3082. http://dx.doi.org/10.1016/j.tet.2013.01.069

35. Bazgir, A.; Ahadi, S.; Ghahremanzadeh, R.; Khavasi, H. R.; Mirzaei, P. Ultrason. Sonochem. 2010, $17,447$. http://dx.doi.org/10.1016/i.ultsonch.2009.09.009

36. Jose, L.; Ruano, G.; Parra, A.; Marzo, L.; Yuste, F.; Mastranzo, V. M Tetrahedron 2011, 67, 2905. http://dx.doi.org/10.1016/j.tet.2011.02.060

37. Ruiz, E.; Rodriguez, H.; Coro, J.; Salfran, E.; Suarez, M.; Martinez-Alvarez, R.; Martin, N. Ultrason. Sonochem. 2011, 18, 32.

http://dx.doi.org/10.1016/j.ultsonch.2010.04.009

38. Said, K.; Moussaoui, Y.; Kammoun, M.; Salem, R. B. Ultrason. Sonochem. 2011, 18, 23. http://dx.doi.org/10.1016/j.ultsonch.2010.04.007

39. Cella, R.; Stefani, H. A. Tetrahedron 2009, 65, 2619. http://dx.doi.org/10.1016/j.tet.2008.12.027

40. Fan, G.; Luo, S.; Wu, Q.; Fang, T.; Li, J.; Song, G. RSC Adv. 2015, 5, 56478. http://dx.doi.org/10.1039/C5RA07431B

41. Moghaddam, F. M.; Kiamehr, M.; Khodabakhshi, M. R.; Javan, M. J.; Fathi, S.; Villinger, A.; Iaroshenko, V. O.; Langer, P.; Helv. Chim. Acta 2013, 96, 2103. 
42. Moghaddam, F. M.; Khodabakhshi, M. R.; Kiamehr, M.; Ghahremannejad, Z. Tetrahedron Lett. 2013, 54, 2685.

http://dx.doi.org/10.1016/i.tetlet.2013.03.070

43. Kiamehr, M.; Moghaddam, F. M.; Gormay, P. V.; Semeniuchenko, V.; Villinger, A.; Langer, P.; laroshenko, V. O. Tetrahedron 2012, 68, 9685.

http://dx.doi.org/10.1016/j.tet.2012.09.059

44. Kiamehr, M.; Moghaddam, F. M. Tetrahedron Lett. 2009, 50, 6723.

http://dx.doi.org/10.1016/j.tetlet.2009.09.106

45. Moghaddam, F. M.; Kiamehr, M. Monatsh Chem 2010, 141, 1333.

http://dx.doi.org/10.1007/s00706-010-0406-1

46. Ziarani, G. M.; Gholamzadeh, P.; Lashgari, N. Hajiabbasi, P. Arkivoc 2013, (i), 470. http://dx.doi.org/10.3998/ark.5550190.p008.074

47. Sun, L.; Tran, N.; Tang, F.; App, H.; Hirth, P.; McMahon, G.; Tang, C. J. Med. Chem. 1998, 41, 2588. http://dx.doi.org/10.1021/jm980123i

48. Andreani, A.; Burnelli, S.; Granaiola, M.; Leoni, A.; Locatelli, A.; Morigi, R.; Rambaldi, M.; Varoli, L.; Kunkel, M. W. J. Med. Chem. 2006, 49, 6922.

http://dx.doi.org/10.1021/im0607808

49. Crystallographic data for $\mathbf{8 i}$ have been deposited in the Cambridge Crystallographic Data Centre with the deposition number CCDC 827753. Copies of data can be obtained free of charge via www.ccdc.cam.ac.uk/conts/retrieving.html (or from the Cambridge Crystallographic Data Centre, 12 Union Road, Cambridge, CB21EZ, UK; fax: +44 1223336 033; or e-mail: deposit@ccdc.cam.ac.uk).

50. Amornraksa, K.; Grigg, R.; Gunaratna, H. Q. N.; Kemp, J.; Sridharan, V. J. Chem. Soc., Perkin Trans. 1987, $1,2285$.

http://dx.doi.org/10.1039/P19870002285 